\title{
COLECTA Y ESTABLECIMIENTO DE ANONÁCEAS EN EL SALVADOR'
}

\author{
Eduardo Cruz ${ }^{2}$, Héctor Deras ${ }^{2}$
}

\section{RESUMEN}

Colecta y establecimiento de anonáceas en EI Salvador. El Centro Nacional de Tecnología Agropecuaria y Forestal (CENTA), a través de la Unidad de Recursos Fitogenéticos, inició en 1998 un diagnóstico geográfico de especies de anonáceas, con el objetivo de explorar, recolectar, caracterizar y establecer colecciones de campo en las Estaciones Experimentales. Las características evaluadas para frutos fueron: peso, largo y diámetro, número de semillas, textura de cáscara y pulpa; forma de fruto y cárpelos, daño por plagas y enfermedades y un análisis bromatológico. Como resultado de este trabajo se estableció la colección de anonáceas con las siguientes especies: squamosa, cherimola, reticulata, muricata, purpurea, diversifolia, holosericea y glabra. En la caracterización morfológica preliminar Annona muricata obtuvo un promedio de 175 semillas/fruto y 1,319 $\mathrm{g}$ en peso del fruto. A. cherimola con pulpa muy arenosa y carpelos no prominentes. A. squamosa con pulpa dulce, blanca y cárpelos muy prominentes. De la caracterización bromatológica se determinó que Annona muricata dio contenidos de humedad superiores a $82,8 \%$; fibra cruda de $1,84 \%$ y fósforo con 367 ppm. En Annona diversifolia con menor grasa $0,01 \%$. La mayor incidencia por plagas en frutos y semillas fue causado por el perforador Bephrateloides sp. La antracnosis (Colletotrichum sp.) en frutos y follaje fue la enfermedad más observada.

\begin{abstract}
Collection and establishment of annonaceae in EI Salvador. The national center of agricultural and forest technology (CENTA), through its unit of phytogenetic resources, under took a collecting survey of annonacea species in 1998, with the objective of exploring, collect, characterize and establish collections in the experimental stations. This survey was carried out at a national level visiting properties and markets. Evaluated traits for fruits were: weight, lenght and diameter, number of seeds, texture of shell and pulp, form of fruit and carpels, damage for pests and diseases. It also was carried out a bromatological analysis. As a result of the survey a collection was established with these species: squamosa, cherimola, reticulata, muricata, purpurea, diversifolia, holosericea and glabra. The morphological characterization Annona muricata showed an average of 175 seeds per fruit and an average weight of 1,319 grames per fruit. A. cherimola showed a very sandy pulp and non-prominent carpels. A. squamosa showed a sweet pulp, white and very prominent carpels. According to the bromatological characterization Annona muricata showed the higher humidity $(82.8 \%), 1.84 \%$ of crude fiber, $367 \mathrm{ppm}$ of phosphorus. Annona diversifolia showed the lowest fat content of $0.01 \%$. The highest pests was caused by Bephrateloides sp. and by Colletotrichum sp. in fruits and foliage.
\end{abstract}

\section{INTRODUCCIÓN}

La familia de las anonáceas está conformada por 80 géneros y cerca de 850 especies (Lawrence, 1951), de los cuales cuatro géneros son los más importantes: Annona, Rollinia, Uvaria y Asiminia (Canizares, 1966). En El Salvador, se reportan nueve especies en estado silvestre y semi cultivadas, siendo la Annona diversifolia y la Annona muricata las de mayor importancia económica. Otras como Annona reticulata, Annona purpurea, Annona cherimola, Annona squamasa, Annona glabra y Annona holosericea, se encuentran en peligro de extinción, con peligro también de perderse características importantes que pueden ser aprovechadas para la preservación de estas especies de alto valor genético y cultural.

Por diversas situaciones los recursos fitogenéticos están en peligro de extinción, debido a la falta de atención y la importancia acerca de la forma de incremen-

1 Presentado en la XLVI Reunión Anual del PCCMCA, Puerto Rico, 2000.

2 Centro Nacional de Tecnología Agropecuaria (CENTA). El Salvador. 
tarlos y conservarlos, a tal grado de formar parte importante de los programas de investigación y como fuente de material genético, para trabajos de selección y propagación vegetativa.

En El Salvador, se reportan nueve especies de anonáceas en estado silvestre y semi cultivada, como son: A. diversifolia, A reticulata, A. purpurea, A.cherimola y A. holoserica.

Annona diversifolia (Ilama), frutos de pulpa blanca o rosada y semillas oblongas de color café lustroso. Las semillas de Ilama, están inactivas por varias semanas o meses después de que el fruto madura, tiempo durante el cual germinan pobremente o no germinan del todo (Cambell y Popenoe, 1967).

En El Salvador y Guatemala, la Anona diversifolia es llamada anona blanca (Popenoe, 1920). En Florida E.U, ha sido introducida de El Salvador (Campbell, 1970). Es originaria de las montañas y laderas del Suroeste de México, El Salvador y Guatemala, a alturas de $600 \mathrm{msnm}$.

Annona reticulata con frutos de cáscara color amarillo y anaranjado, pulpa color crema y semillas negras brillantes.

Annona glabra, frutos de pulpa insípida, color anaranjado, semillas café lustroso.

Annona purpurea, fruto grande con protuberancias piramidales de pulpa anaranjada y fibrosa.

Annona muricata, fruto con pulpa blanca, ácida o dulce y semillas negras lustrosas. La importancia económica de ésta recae en la pulpa, la cual se puede consumir en estado fresco o puede ser procesado para preparar néctares, conservas, helados,etc (Sánchez Nieva et al. 1970).

Annona cherimola, frutas de pulpa blanca, de sabor dulce, muy arenosa, de forma ovoide a globosa y superficie lisa. La Annona cherimola posee un alto valor nutritivo y alto contenido de fibra, vitamina $\mathrm{C}$ y Niacina; por lo que constituye un material genético importante para trabajos de hibridación, selección y propagación vegetativa.

Annona squamosa, frutos ovoides con carpelos prominentes, de pulpa blanca y semillas negras lustrosas. La Annona squamosa germina de 20 a 30 días después de la siembra, con 90 a $95 \%$ de germinación, cuando se almacenan en estado fresco (Miraflores, 1915), en contraste con la germinación de Annona chirimola, y An- nona diversifolia que es altamente variable en un intervalo de 30 a $80 \%$ (Campbell y Popenoe, 1967).

Annona holosericea, frutos pequeños, redondos, con protuberancias piramidales y superficie amarilla aterciopelada.

Según Nidiel (1981) y (Ponce, 1981), Bephratelloide cubensis, es una de las principales plagas de la Annona muricata en el estado de Veracruz, México, causando pérdidas en la producción de aproximadamente un $25 \%$.

Según el anuario estadístico (1997-1998) del Ministerio de Agricultura y Ganadería (MAG), El Salvador, no se reportan datos de importación y de exportación de anonas.

\section{MATERIALES Y MÉTODOS}

El estudio se inició a finales de 1997 en los 14 departamentos de El Salvador. Se efectuaron giras de exploración a propiedades de productores y mercados de la localidad, en las que se recopiló la información de las especies de anonáceas, su localización y distribución en las diferentes zonas. Se empleó una boleta de inventario que incluye datos pasaporte, datos de recolección, información etnobotánica y características de frutos, semillas y follaje. Las especies en estudio fueron las siguientes: Annona muricata, Annona squmosa, Annona reticulata, Annona cherimola, Annona purpurea, Annona diversifolia, Annona glabra y Annona holosericea.

El Centro Nacional de Tecnología Agropecuaria y Forestal (CENTA), a través de la Unidad de Recursos Fitogenéticos, inició en 1998 la recolecta de anonáceas, caracterización y establecimiento una colección en campo, en las estaciones experimentales del CENTA. Con las primeras exploraciones se recolectaron tres especies: Annona diversifolia, Annona muricata y Annona reticulata (Cruz, Deras 1988).

Los caracteres cualitativos evaluados fueron: color y textura de cáscara y pulpa, forma de carpelos y de frutos, sabor, jugosidad de los frutos, color del haz y envés de la hoja. Los caracteres cuantitativos fueron peso (g), longitud $(\mathrm{cm})$, diámetro $(\mathrm{cm})$, número de semilla y características bromatológicas como: humedad (\%), proteínas (\%), grasa (\%), cenizas (\%), Fósforo (ppm), Calcio (ppm), Potasio (ppm).

La información se analizó por estadística descriptiva para valores mínimo, máximo y medias. 
Cuadro 1. Especies del género Annona reportadas, información etnobotánica y su distribución en El Salvador. 1999.

\begin{tabular}{|c|c|c|}
\hline Nombre científico & Nombre común & Distribución \\
\hline Annona squamosa & Poshta, bayunca, anona montés & La Unión, Chalatenango, La Libertad. \\
\hline Annona cherimola & Anona, chirimuya, chirimoya & Chalatenango \\
\hline Annona muricata & Guanaba, guanábana & $\begin{array}{l}\text { Santa Ana, Ahuachapán, Sonsonate, La } \\
\text { Libertad, Cuscatlán, San Salvador, La Paz, } \\
\text { Usulután, San Miguel, Morazán, Chalatenango }\end{array}$ \\
\hline Annona purpurea & Sincuya, Suncuya & $\begin{array}{l}\text { Santa Ana, Chalatenango, La Libertad, } \\
\text { Cuscatlán, San Miguel, La Unión, Usulután, } \\
\text { Sonsonate, Ahuachapán, La Paz. }\end{array}$ \\
\hline Annona reticulata & $\begin{array}{l}\text { Anona colorada, Anona montés } \\
\text { Anona corazón de buey }\end{array}$ & $\begin{array}{l}\text { La Libertad, La Paz, Sonsonate, San Vicente, } \\
\text { San Miguel, Ahuachapán. }\end{array}$ \\
\hline Annona glabra & Anona de manglar & La Libertad \\
\hline Annona holosericea & Suncuyita, Chirimuya & La Unión, San Miguel, La Libertad, \\
\hline Annona diversifolia & Anona blanca, de castilla, Caribe o rosada & $\begin{array}{l}\text { Santa Ana, Ahuachapán, Sonsonate, } \\
\text { Chalatenango, La Libertad, San Salvador, } \\
\text { Cuscatlán, Cabañas, San Vicente, La Paz, } \\
\text { Usulután, Morazán, San Miguel, La Unión. }\end{array}$ \\
\hline
\end{tabular}

\section{RESULTADOS}

En el Cuadro 1, se presentan las especies del género Annona en El Salvador y su distribución en los departamentos en que fueron localizadas, siendo estas: Annona muricata, Annona reticulata, Annona purpurea, Annona squamosa, Annona cherimola, Annona glabra, Annona diversifolia, Annona holosericea. Además, la diversidad de nombres comunes con los que las especies son conocidas principalmente por la apariencia del fruto.

En el Cuadro 2, se muestran las características morfológicas de cinco especies de anonáceas. Annona squamosa, mostró carpelos muy prominentes y pulpa arenosa; Annona cherimola y Annona reticulata, textura de cáscara lisa y carpelos no prominentes. El mayor promedio en peso de fruto es Annona muricata con 1319 g, seguido de Annona diversifolia con 739 g. El número de semillas por fruto es mayor en Annona ти- ricata con 175 semillas y Annona reticulata, con 80 semillas. Annona cherimola, con textura de pulpa más arenosa y menos en Annona squamosa. En cuanto al rango de adaptabilidad, Annona cherimola se localizó entre los 1000-1800 msnm., mientras que el resto de especies varió entre los 30-800 msnm.

En el Cuadro 3, se muestra el análisis bromatológico de la pulpa de Annona cherimola, Annona muricata y Annona diversifolia.

En Annona muricata, se mostró mayor en humedad con $82,8 \%$, proteína $2,18 \%$, fibra cruda con $1,84 \%$. Annona cherimola, mostró mayor contenido en Fósforo con $367 \mathrm{ppm}$ y grasa con $0,44 \%$. Annona diversifolia, mostró menor contenido en grasa con $0,01 \%$ en relación a Annona cherimola.

En el Cuadro 4, se muestran los daños por plagas y enfermedades más importantes en especies de anonáceas.

Cuadro 2. Características morfológicas de cinco especies de Annona, El Salvador 1999,

\begin{tabular}{|c|c|c|c|c|c|c|c|c|}
\hline Especie & Peso (g) & $\begin{array}{l}\text { Número } \\
\text { semillas }\end{array}$ & $\begin{array}{l}\text { Forma } \\
\text { fruto }\end{array}$ & $\begin{array}{c}\text { Textura } \\
\text { pulpa }\end{array}$ & $\begin{array}{l}\text { Color } \\
\text { pulpa }\end{array}$ & $\begin{array}{l}\text { Textura } \\
\text { cáscara }\end{array}$ & $\begin{array}{c}\text { Forma } \\
\text { de Carpelos }\end{array}$ & m.s.n.m \\
\hline Annona squamosa & 322 & 64,6 & ovoide-esférico & arenosa blanda, dulce & blanca & áspera & Muy prominente & $200-700$ \\
\hline Annona cherimola & 453 & 52,4 & ovoide & muy arenosa, blanda, dulce & blanca & lisa & No prominente & $1000-1800$ \\
\hline Annona muricata & 1,319 & 175,0 & ovoide-elipsoidal & $\begin{array}{l}\text { arenosa, blanda, ácida o dulce } \\
\text { y equinados }\end{array}$ & blanca & lisa & No prominente & $200-700$ \\
\hline Annona reticulata & 628 & 80,0 & ovoides-esférico & blanda, dulce & blanca & lisa & No prominente & $30-700$ \\
\hline Annona diversifolia & 739 & 69,6 & ovoide-elipsoidal & blanda, dulce, rosada & blanca o & áspera & $\begin{array}{l}\text { Prominente y } \\
\text { no prominente }\end{array}$ & $100-800$ \\
\hline
\end{tabular}


Cuadro 4. Daños observados por plagas y enfermedades en especies de Annona. El Salvador, 1999.

\begin{tabular}{|c|c|c|c|}
\hline Especie & Plaga & Enfermedad & Daño \\
\hline A. muricata & Bephrateloides sp. Perforación de frutos y semilla & Colletotrichum sp. & Momificación de frutos. \\
\hline A. holosericea & & Colletotrichum sp. & $\begin{array}{l}\text { Manchas necróticas con halo } \\
\text { amarillo en el haz de la hoja. }\end{array}$ \\
\hline A. diversifolia & Bephrateloides sp. Perforación de frutos & $\begin{array}{l}\text { Colletotrichum sp. } \\
\text { Botriodiplodia sp. } \\
\text { Macrophoma sp. }\end{array}$ & $\begin{array}{l}\text { Momificación de frutos y } \\
\text { necrosis en follaje. }\end{array}$ \\
\hline A. reticulata & Bephrateloides sp. Perforación de frutos y semilla & Colletotrichum sp. & $\begin{array}{l}\text { Momificación de frutos y } \\
\text { necrosis en el follaje. }\end{array}$ \\
\hline
\end{tabular}

Para Annona muricata y Annona reticulata, se observó el mayor daño por el barrenador perteneciente al género Bephratelloide, tanto en frutos como en semi-

Cuadro 3. Análisis bromatológico de la pulpa de tres especies de Annona. El Salvador, 1999.

\begin{tabular}{lccc}
\hline $\begin{array}{c}\text { Análisis } \\
\text { (base húmeda) }\end{array}$ & $\begin{array}{c}\text { Annona } \\
\text { cherimola }\end{array}$ & $\begin{array}{c}\text { Annona } \\
\text { muricata }\end{array}$ & $\begin{array}{c}\text { Annona } \\
\text { diversifolia }\end{array}$ \\
\hline Humedad (\%) & 67,8 & 82,8 & 79,6 \\
Proteínas (\%) & 1,78 & 2,18 & 1,31 \\
Grasa (\%) & 0,44 & - & 0,01 \\
Ceniza (\%) & 0,91 & 1,24 & 1,26 \\
Fibra cruda(\%) & 1,28 & 1,84 & 0,97 \\
Fósforo (ppm) & 367 & 301 & 300 \\
Calcio (ppm) & 128 & 228 & - \\
\hline
\end{tabular}

1las. La antracnosis causada por el hongo Colletotrichum sp., es la enfermedad más común que se encontró afectando considerablemente a frutos y follaje, disminuyendo significativamente la cosecha. Las especies más afectadas son: Annona muricata, Annona diversifolia, y Annona reticulata.

En la Figura 1 se muestra la distribución geográfica de las especies de Anonáceas en El Salvador.

\section{CONCLUSIONES}

La información etnobotánica mostró que las especies: Annona diversifolia, Annona muricata, Annona squamosa, Annona cherimola y Annona purpurea, poseen mayor valor comercial entre los pobladores de la zona.

Annona cherimola, se caracterizó por la pulpa de color blanco, dulce, muy arenosa, de cáscara lisa y sin cárpelos visibles; mayor contenido de fósforo y un rango de adaptación entre 1000 a 1800 msnm.

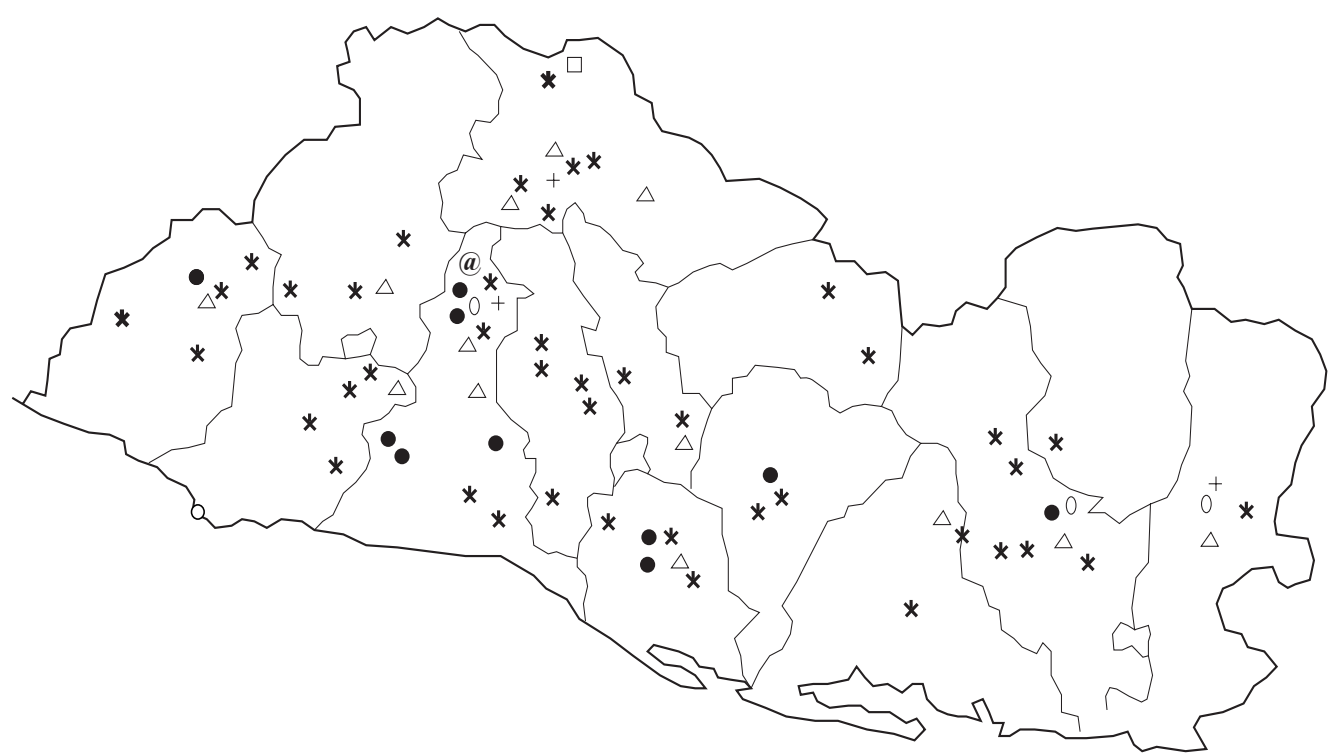

Figura 1. Distribución geográfica de especies de anonáceas en El Salvador, 1999 A. squamosa (+); A.cherimola $\square$ );

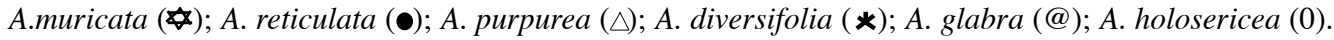


Annona squamosa, se caracterizó por frutos esféricos u ovoides, pulpa blanca arenosa, cáscara áspera y cárpelos muy prominentes.

El mayor daño en frutos y semillas, fue causado por el perforador del fruto Bephrateloide $s p$; en cuanto a enfermedades la de mayor importancia económica fue antracnosis causada por Colletotrichum sp.

\section{RECOMENDACIONES}

Continuar con el diagnóstico ecogeográfico de anonáceas, a fin de rescatar y conservar las especies en peligro de extinción.

Coordinar con Instituciones y Organismos Nacionales e Internacionales; actividades que conlleven a la ejecución de trabajos de selección y mejoramiento de éstas especies.

Coordinar con los programas de Agroindustria y Frutales, investigaciones sobre el potencial de uso de la pulpa de anonáceas, así como, el mejoramiento genético de las especies.

\section{LITERATURA CITADA}

CASILLAS ESTRADA, A; MARROQUÍN ANDRADE, L. 1994. Caracterización de Ilama (Annona diversifolia). Depto. Fitotecnia. Universidad Autónoma, Chapingo, México, pp.197-199.

CONGRESO INTERNACIONAL DE ANNONÁCEAS. 1997. Memoria. Chapingo, México. 264 p.

INSTITUTO INTERNACIONAL DE RECURSOS FITOGENÉTICOS (IPGRI). 1999. Boletín de las Américas. Cali, Colombia. 5(1), p. 2-3.

MARTÍNEZ, V.A; MARTÍNEZ, T.E. 1999. Distribución geográfica de anonas en Guatemala. Universidad de San Carlos de Guatemala, Facultad de Agronomía. 9 p.

MARROQUÍN, L.M.; HERNÁNDEZ, R.R; MARTÍNEZ, J.S.; VERGARA, M.A. 1997. Tratamientos pregerminativos en semillas de Ilama (Annona diversifolia Saff). Depto. Suelos de la Universidad Autónoma de Chapingo, Chapingo, México. pp. 62-64.

MINISTERIO DE AGRICULTURA Y GANADERÍA. Dirección General de Economía Agropecuaria. Anuario de Estadísticas. 1997-1998. Ed. 37. San Salvador, El Salvador. pp. 53-56.

VILLACORTA, MONZÓN, R. Las anonas de El Salvador. Jardín Botánico La Laguna, San Salvador, El Salvador. pp.7-9. 